\title{
Surgical Repair of Coronary Artery Aneurysm After Percutaneous Coronary Intervention
}

\author{
Hiroichiro Yamaguchi, MD; Hideto Yamauchi, MD; \\ Takafumi Yamada, MD; Tsuneo Ariyoshi, MD
}

\begin{abstract}
Two cases of coronary artery aneurysm after percutaneous coronary intervention are presented. In both cases, follow-up coronary angiography revealed an expanding saccular aneurysmal formation and restenosis or a new lesion. Consequently, surgical repair and revascularization were indicated. The intraoperative angioscope was used to identify the aneurysm, which had not been visible on the heart surface in one case. Its optic fiber light was a useful guide, but little information was obtained from direct vision because of the small caliber of the angioscope. Plication of the aneurysm and bypass grafting distal to the aneurysm were performed. A whitish and thick-walled aneurysm was easily found in one case, in which ligation of the aneurysm was attempted with added distal bypass. The postoperative course was uneventful in both cases, and follow-up angiography showed disappearance of the aneurysm with patent grafts. There was a fragile thrombus inside both the aneurysmal sacs, which might have caused myocardial infarction and, therefore, justified the surgical repair of the aneurysm with concomitant revascularization. (Jpn Circ J 2001; 65: 52-55)
\end{abstract}

Key Words: Coronary artery aneurysm; Coronary artery bypass grafting; Coronary stent implantation; Percutaneous transluminal coronary angioplasty

$\mathbf{P}$ ercutaneous transluminal coronary angioplasty (PTCA) occasionally causes the unexpected adverse effect of a coronary artery aneurysm, which is prone to rupture and thrombose and therefore requires repair! With the increasing use of new devices, and newer ones appearing in quick succession, an increase in the frequency of this complication is expected. We describe here 2 cases of coronary artery aneurysm after PTCA, and their successful surgical repair.

\section{Case Reports}

Case 1

A 60-year-old man developed severe post-infarction angina in December 1992. Coronary angiography (CAG) revealed $99 \%$ stenosis of the proximal left anterior descending coronary artery (LAD) and PTCA was performed, after which the residual stenosis was $50 \%$. The patient was subsequently asymptomatic. However, the follow-up CAG after 1 month showed a small coronary artery aneurysm at the site of the previous PTCA. In April 1993, chest pain recurred and CAG showed an enlarging aneurysm and 75\% restenosis of LAD and a new 75\% stenosis of the distal circumflex coronary artery (CX) (Fig 1). Coronary artery bypass grafting $(\mathrm{CABG})$ and surgical repair of the aneurysm were therefore indicated.

Surgery was performed in May 1993. The aneurysm was not visible on the heart surface, so we used a $0.5-\mathrm{mm}$ angioscope (Olympus Optical Co, Ltd, Japan) to determine the

(Received June 28, 2000; revised manuscript received August 28, 2000; accepted August 30, 2000)

Department of Cardiovascular Surgery, Oita Prefectural Hospital, Oita, Japan

Mailing address: Hiroichiro Yamaguchi, MD, Department of Cardiovascular Surgery, Oita Prefectural Hospital, 476 Bunyo, Oita-city, Oita 870-8511, Japan site of the aneurysm. The angioscope was inserted through a small hole in the distal LAD, but, actually, little helpful information on the aneurysm was obtained through direct vision because of the scope's small caliber. The aneurysmal region of the proximal LAD, which was covered with thick epicardial fat, was identified and exposed through the guidance of the optic fiber light and the angiographical finding.

The aneurysm was dissected longitudinally and plicated after removal of the mural thrombus. In addition, double CABG to the initial hole in the distal LAD with the left internal mammary artery and to the CX with the saphenous vein was performed.

The postoperative course was uneventful and CAG at 1 month after the operation showed that the aneurysm had disappeared and both grafts were patent (Fig 1). We were not able to distinguish pseudoaneurysm from a true one histopathologically from the surgical specimen.

\section{Case 2}

A 76-year-old man was admitted because of chest pain in October 1999. CAG showed an occlusion of the proximal LAD, and an ACS Multi-Link stent $(3.0 \times 15 \mathrm{~mm})$ was implanted after PTCA. The target lesion had no residual stenosis, but the follow-up CAG 4 months after the first intervention showed restenosis at the distal site of the stent. A second PTCA using a cutting balloon $(3.0 \times 15 \mathrm{~mm})$ was performed and extravasation of the contrast media was demonstrated at that time. Inflation of another balloon prevented further leakage, and the patient was discharged without subsequent complication. In May 2000, chest pain recurred and CAG demonstrated a $9.0 \times 5.0 \mathrm{~mm}$ coronary artery aneurysm and restenosis at the proximal site of the stent (Fig 2). Therefore, revascularization and surgical repair of the aneurysm was required.

At operation on May 30, 2000, the aneurysm was easily found on the surface of the heart, being whitish, and elastic 

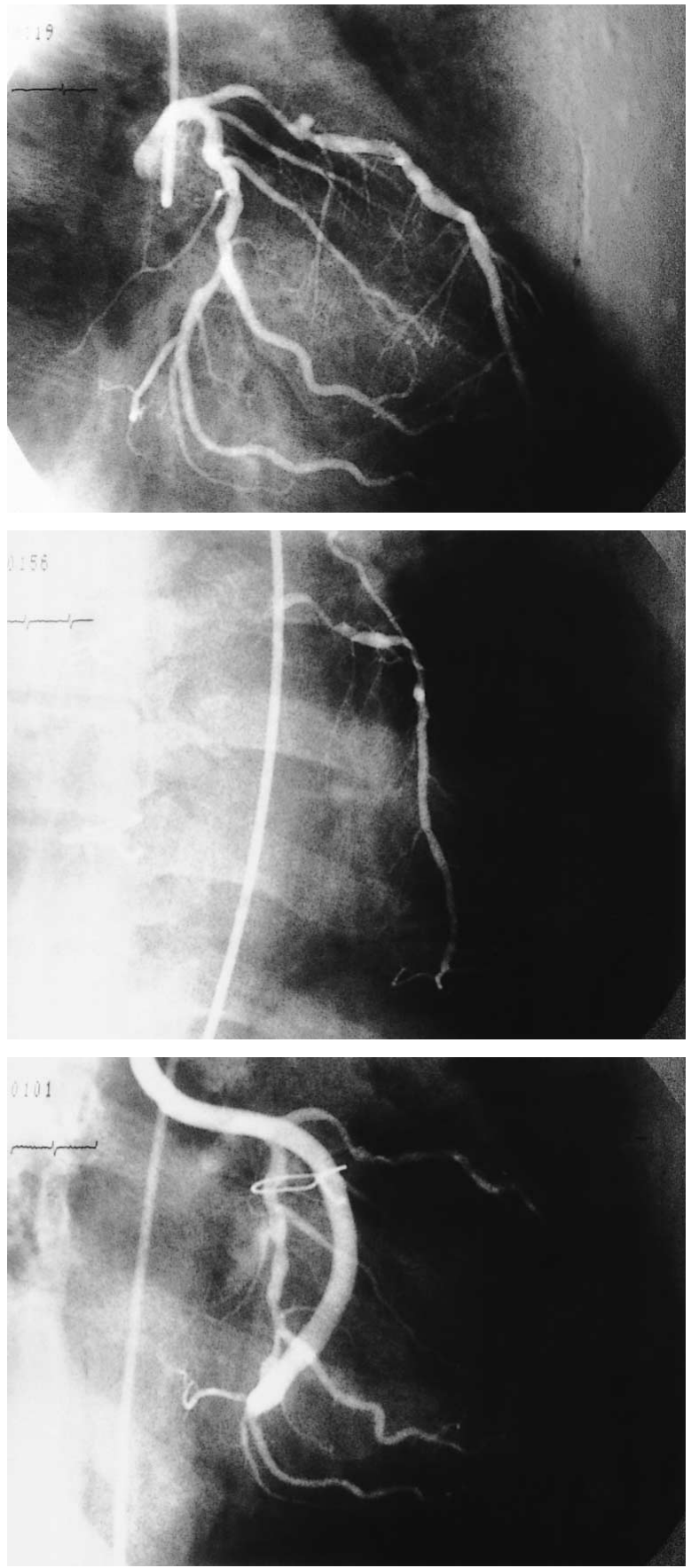

Fig 1. Coronary angiogram in case 1. (Upper) Preoperative left coronary artery angiogram. Coronary artery aneurysm at the proximal site of LAD and stenoses of LAD and CX are shown. (Middle) Postoperative left internal mammary artery angiogram. (Lower) Postoperative vein graft angiogram. Disappearance of the aneurysm and patent grafts are shown. LAD, left anterior descending coronary artery; CX, circumflex coronary artery.

hard to palpation. Under cardiac arrest with cardioplegia, we could also identify the aneurysm intravascularly using a 1.4-mm angioscope (Olympus Optical Co, Ltd), which was inserted from the distal LAD. The aneurysm was dissected longitudinally and the thrombus inside the sac was removed (Fig 3). Because it was difficult to identify the healthy intima distinctly, the aneurysm was ligated instead of plicated. Finally the left internal mammary artery was anastomosed
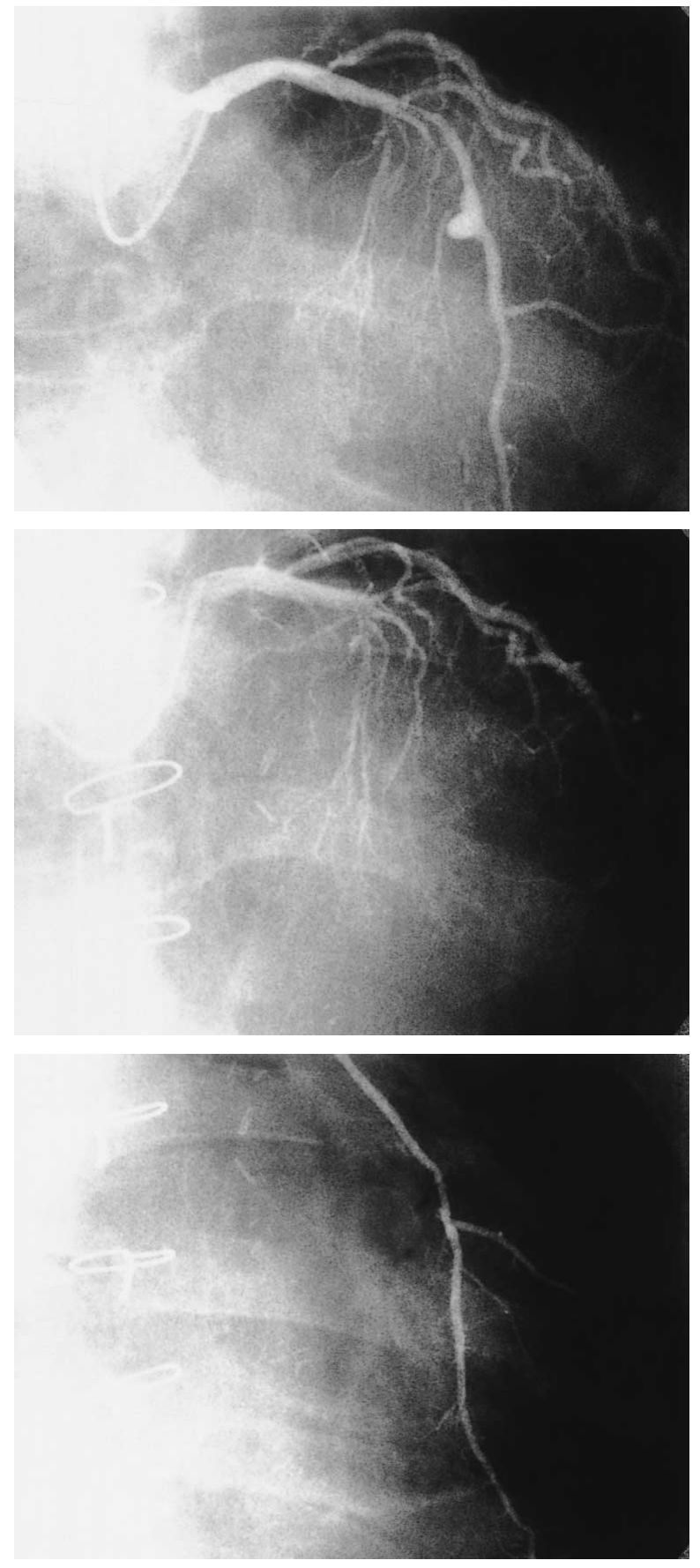

Fig 2. Coronary angiogram in case 2. (Upper) Preoperative left coronary artery angiogram. Coronary artery aneurysm at the proximal site of LAD and stenosis of LAD are shown. (Middle) Postoperative left coronary artery angiogram. Disappearance of the aneurysm and preserved major septal branches are shown. (Lower) Postoperative left internal mammary artery angiogram. Patent graft to distal LAD is shown. LAD, left anterior descending coronary artery.

\section{to the LAD.}

The patient has had a good postoperative course. The CAG at 3 weeks after the operation showed the defect, but there was good perfusion to the distal LAD through the graft (Fig 2). The resected aneurysmal wall contained the media, so the diagnosis of pseudoaneurysm was not obtained histopathologically. 


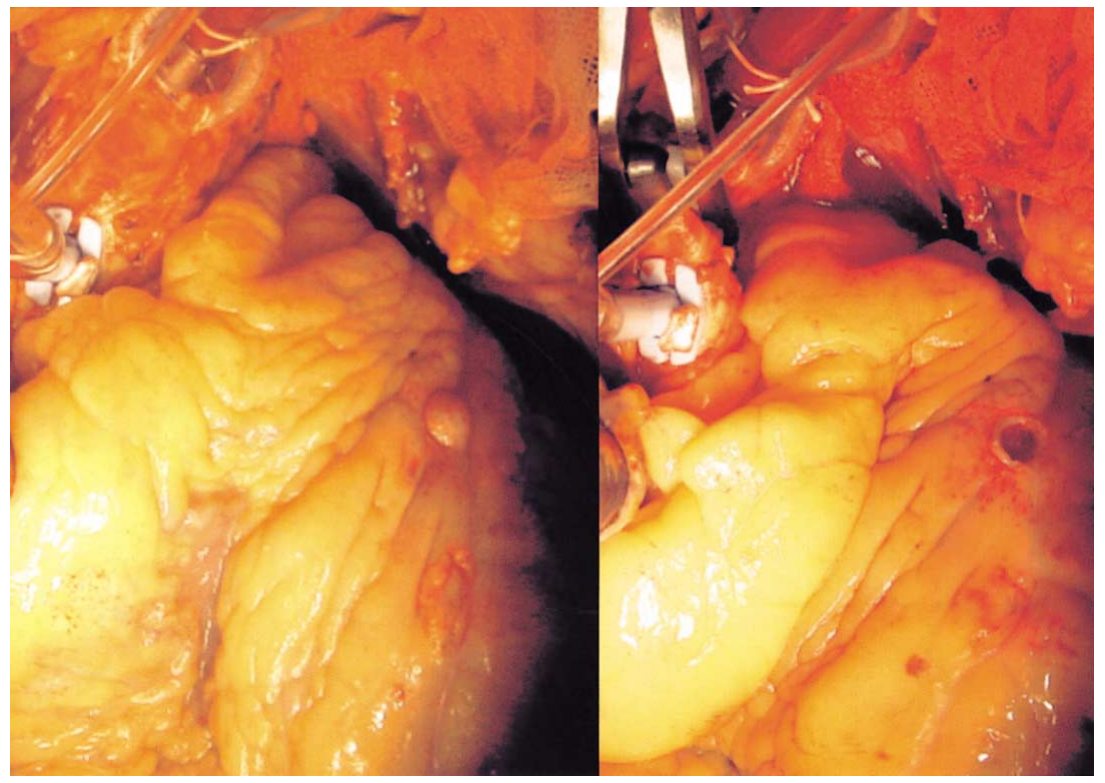

Fig 3. Intraoperative findings in case 2. (Left) A whitish, elastic hard mass can be seen along the LAD on the heart surface. (Right) Fragile fresh thrombus in the aneurysm with thick aneurysmal wall. LAD, left anterior descending coronary artery.

\section{Discussion}

PTCA-induced pseudoaneurysm of the coronary artery is caused either by incomplete healing after perforation or rupture of the coronary artery occurring at the time of the coronary intervention. According to a report by Ajluni et al, perforation associated with conventional PTCA occurred in $0.14 \%$ of cases? However, aneurysm of the coronary artery requiring surgical repair, except in emergency operations for acute heart failure because of rupture, is not very familiar to most surgeons. A recent review by Dralle et al reported that there were only 74 cases of coronary aneurysm associated with percutaneous catheter interventions reported in the English-language medical literature up to 1993 ? However, the more recent rapid development and spread of new procedures such as coronary atherectomy, in which the possibility of pseudoaneurysm developing is thought to be higher than that of conventional PTCA2 may increase the incidence. Therefore, we need to establish a strategy for the management of coronary artery aneurysm associated with percutaneous coronary intervention.

Whether an aneurysm relevant to the percutaneous coronary intervention in itself can be indication for surgery is the contentious issue. In the review of 74 cases of coronary artery aneurysm, only 16 of the patients involved had undergone operation, and 14 of those had another indication for repair? Cohen et al reported a case where, despite undergoing surgical dissection and being left in the coronary circulation, the aneurysm did not expand during 2 months of follow-up4 We performed the 2 present operations both because of the aneurysm and because of restenosis or another new lesion.

The type of aneurysm may be an important factor in the indication for surgical repair of a coronary artery aneurysm. The less likely formation of a true aneurysm, resulting from the healing of a deep dissection, after PTCA is presumably the reason for the low morbidity and mortality5 On the other hand, the fibrous wall of a pseudoaneurysm resulting from traumatic rupture is not in continuity with the structure of the adjacent vessel wall, and so has the potential for progressive enlargement or rupture. The aneurysm type in case 1 was unknown, because no evidence of pseudoaneurysm was obtained clinically or pathologically. However, enlargement of the aneurysm and the restenosis and appearance of a new lesion were the determinants for surgical exploration. It was especially apparent in case 2 that the primary coronary perforation induced an expanding aneurysm despite not obtaining pathological evidence of a pseudoaneurysm. The pathological diagnosis that it was not pseudoaneurysm was based on the surgical specimen contained the media. We assume that incomplete resection of the tissue demonstrating discontinuity with the structure of the vessel wall was responsible for pathological diagnosis in case 2.

Differentiation of the aneurysm type is important. Preoperative use of intravascular ultrasound may be helpful for the diagnosis and surgical indication? In the end, the aneurysms in both the present cases were thick-walled and did not threaten to rupture; instead, they expanded to nearly $1 \mathrm{~cm}$ during 3 months as demonstrated angiographically. However, each aneurysm had fragile fresh thrombus inside its sac and as this is regarded as one of the causes of myocardial infarction or death, we believe this justified repair of the aneurysm in addition to CABG.

Concerning the surgical procedure for treatment of the aneurysm, the choices are (i) leaving the aneurysm alone, but employing isolated revascularization, (ii) ligation, or (iii) plication of the aneurysm with concomitant revascularization? Attempted ligation of LAD aneurysms may inadvertently sacrifice important septal perforators, and may demand larger flow reserve of the bypass conduit to the distal coronary immediately, in the case of only a mild stenosis of the proximal site of the aneurysm. Consequently, hypoperfusion syndrome may occur4 Dralle et al recommended plication of the aneurysm so as to maintain some antegrade coronary flow in the perioperative period, thus decreasing the immediate flow demands on the graft, 3 and this is the technique we employed in case 1 .

The intraoperative angioscope was helpful for identifying the location of the aneurysm, although it was hard to observe the aneurysm intravascularly. The very small caliber of the angioscope was thought to be responsible for this difficulty in case 1 . In case 2 , the thrombus formation and obscured intimal layer inside the aneurysm compelled us to abandon plication and attempt ligation. Once incised the aneurysm 
was clamped with tourniquets at both the proximal and distal sites until cessation of cardiopulmonary bypass, and it was doubly ligated after we confirmed stable hemodynamics without the pump. Preoperative CAG showed no visible septal branch originating from the aneurysmal lesion, and no evidence of intraoperative ischemia was found postoperatively.

The catheter interventional management using an autologous vein-covered stent is also an attractive approach to the repair of an aneurysm?, but the number of case reports is limited and long-term results still remain unclear. Surgical approach is thought to be safer and more reliable for repair of a pseudoaneurysm. We believe that an enlarging pseudoaneurysm, suggested clinically or radiologically, or any type of aneurysm after percutaneous coronary intervention concomitant with a coronary artery stenotic lesion indicates the need for surgical repair. We performed surgery under cardiac arrest with cardiopulmonary bypass in the present cases, but repair of the aneurysm and revascularization on the beating heart could be an option in selected patients.

\section{References}

1. Anabtawi IN, de Leon JA: Arteriosclerotic aneurysms of the coronary arteries. J Thorac Cardiovasc Surg 1974; 68: 226-228

2. Ajluni SC, Glazier S, Blankenship L, O'Nell WW, Safian RD: Perforations after percutaneous coronary interventions: Clinical, angiographic, and therapeutic observations. Cathet Cardiovasc Diagn 1994; 32: 206-212

3. Dralle JG, Turner C, Hsu J, Replogle RL: Coronary artery aneurysms after angioplasty and atherectomy. Ann Thorac Surg 1995; 59: $1030-$ 1035

4. Cohen AJ, Banks A, Cambier P, Edwards FH: Post-atherectomy coronary artery aneurysm. Ann Thorac Surg 1992; 54: 1216-1218

5. Bal ET, Plokker HWT, van den Berg EMJ, Ernst SMPG, Mast EG, Gin RMTJ, et al: Predictability and prognosis of PTCA-induced coronary artery aneurysms. Cathet Cardiovasc Diagn 1991; 22: 85-88

6. Berkalp B, Kervancioglu C, Oral D: Coronary artery aneurysm formation after balloon angioplasty and stent implantation. Int $J$ Cardiol 1999; 69: $65-70$

7. Gruberg L, Roguin A, Beyar R: Percutaneous closure of a coronary aneurysm with a vein coated stent. Cathet Cardiovasc Diagn 1998; 43: $308-310$ 